\title{
Modeling High-Temperature Superconductivity: Correspondence at Bay?*
}

\author{
Stephan Hartmann
}

August 13, 2007

\begin{abstract}
How does a predecessor theory relate to its successor? According to Heinz Post's General Correspondence Principle, the successor theory has to account for the empirical success of its predecessor. After a critical discussion of this principle, I outline and discuss various kinds of correspondence relations that hold between successive scientific theories. I then look in some detail at a case study from contemporary physics: the various proposals for a theory of high-temperature superconductivity. The aim of this case study is to understand better the prospects and the place of a methodological principle such as the Generalized Correspondence Principle. Generalizing from the case study, I will then argue that some such principle has to be considered, at best, as one tool that might guide scientists in their theorizing. Finally I present a tentative account of why principles such as the Generalized Correspondence Principle work so often and why there is so much continuity in scientific theorizing.
\end{abstract}

\section{Introduction}

Philosophers of science provide us with idealized accounts of science. Sadly, however, these accounts often do not work, as the endless series of discussions among philosophers shows. These discussions typically follow a common scheme: In step 1, a philosopher suggests a theory of $X$ (say, explanation, theory change or what have you). In step 2 , other philosophers criticise this account. Some point out internal problems of the account in question, others present a counterexample. Such a counterexample shows that

*Forthcoming in: L. Soler, H. Sankey and P. Hoyningen-Huene (eds.), Rethinking Scientific Change. Stabilities, Rupture, Incommensurabilities? Berlin: Springer 2008. 
the account in question does not always apply and this is taken to effectively refute it alltogether. In step 3, a new universal account is suggested and it is shown, perhaps, that it deals well with the counterexamples of the previous account. But it is soon shown to have other problems. And so on. Philosophers typically come up with universal and all-encompassing accounts, often based on or motivated by some simple and plausible principle which, at the end of the day, almost inevitably fails when confronted with the practice of science.

Scientists, on the other hand, proceed in a diffferent way, which - if successful - combines a bottom-up (i.e. data-driven) methodology with a top-down (i.e. theory-driven) methodology. They construct models which account for a comparably small set of phenomena and do not intend for them to be universal in scope. Models are local, not global or universal, and once a models fails, its domain of applicability is (typically, though not necessarily) restricted and a new model with a (typically, though not necessarily) wider scope is put forward. Most importantly, scientists are aware of the fact that models involve idealizations and that they do not provide final answers to all questions they have about the object or system under investigation. And yet, models serve various purposes in the process of science (explanation, prediction, policy recommendations etc.), and they do so very well, which is arguably one of the reasons why science as a whole is so successful. While some models in science are formulated in the framework of a theory ("models of a theory"), such as classical mechanics, others are constructed in the absence of a model-constraining theory ("phenomenological models"). A stock example of a model of a theory is the model of a pendulum, and Bohr's model of the atom is an example of a phenomenological model (see Frigg and Hartmann (2006)).

I hold that philosophers of science can learn much from scientists. They should be more modest and aim at constructing models, not theories that aim at getting everything right in just one shot. As in science, more general principles might be applied here or there, but their applicability has to be approached critically. Rather than arguing for this point in abstracto, I will present an example from the philosophy of science that indicates how my proposal works. ${ }^{1}$

The philosophical discussion of scientific theory change illustrates my approach very well. Leaving somewhat more fine-grained positions aside, two main accounts of scientific theory change can be identified. On the one hand, there is the traditional cumulative account according to which science progresses by adding more and more details to already existing accounts. This view, which stresses the continuity of scientific theorizing, met serious criticisms when confronted with episodes from the history of science. Inspired by this criticism, Kuhn, Feyerabend and others suggested an account of scientific theory change

\footnotetext{
${ }^{1}$ For more on my views on modeling in philosophy of science, see Hartmann (2007).
} 
that stresses discontinuities in scientific theorizing. ${ }^{2}$ Buzz words like 'scientific revolution' and 'incommensurability' figure prominently in this account, which gives, just like the cumulative view, a universal answer to the question of how scientific theory change works. One universal philosophical account is replaced by another universal account, but both get in trouble when confronted with cases from real science, as scientific theory change involves both continuity (or stability) and discontinuity (or instability).

There is certainly much more continuity in scientific theorizing, even across revolutions, than Kuhn and his followers made us think. And there might be even more continuity in the future. In The Social Construction of What?, Hacking elaborates this point:

[F]uture large-scale instability seems quite unlikely. We will witness radical developments at present unforseen. But what we have may persist, modified and built upon. The old idea that sciences are cummulative may reign once more. Between 1962 (when Kuhn published Structure) and the late 1980s, the problem for philosophers of science was to understand revolution. Now the problem is to understand stability. (Hacking 1999: 85)

So how can the prevalent stability in science be understood philosophically? As the simple cumulative model does not work, an account is needed that stresses, besides the importance of continuities (or stability), the inevitable presence of more or less disruptive discontinuities (or instability) in scientific theorizing. Such an account is hard to come by and I will not present a fully worked out version of it in this contribution. Instead, I will point out a reasonable way for how one should proceed to arrive eventually at such an account. Following the scientist's strategy, I propose to combine a top-down with a bottom-up strategy and will proceed in two steps: First, I will examine examples from real science to obtain an account of the various ways in which scientific theories relate to their predecessors. This step follows a bottom-up strategy. Second, I will try to understand philosophically the prevalent continuitiy (or stability) in scientific theorizing, as pointed out by Hacking and as suggested by my case studies. This step proceeds in top-down fashion, as I will relate the findings of the case study to a more general, though sufficiently flexible, philosophical theory.

To set the scene, I will start with a critical discussion of Heinz Post's Generalized Correspondence Principle (section 2). I will then outline and discuss various kinds of correspondence relations that hold between successive scientific theories (section 3). Section

\footnotetext{
${ }^{2}$ This is not to say that there is no place for continuities in Kuhn's philosophy of science. In The Structure of Scientific Revolutions, Kuhn (1996) devotes a whole chapter to a discussion of "normal science", which is characterized by an accumulation of solved puzzles. Scientific theory change, however, is discontinuous.
} 
4 then looks in some detail at a case study from contemporary physics: the various proposals for a theory of high-temperature superconductivity. The aim of this case study is to understand better the prospects and the place of a methodological principle such as the Generalized Correspondence Principle. Generalizing from the case study, I will then argue that some such principle has to be considered, at best, as one tool that might guide scientists in their theorizing (section 5). Finally, in section 6, I present a tentative account of why principles such as the Generalized Correspondence Principle work so often and why there is so much continuity in scientific theorizing.

\section{Post's General Correspondence Principle}

Post's General Correspondence Principle is a generalization of the quantum mechanical correspondence principle. ${ }^{3}$ This principle played a crucial role for Niels Bohr and others in the process of constructing the new quantum mechanics in the 1920s. It was expected that quantum mechanics would account, within certain limits, for the well-confirmed phenomena of classical physics. The quantum mechanical correspondence principle is however somewhat more complicated, as Radder (1991) has shown. The latter consists of various interrelated parts which I will not discuss here. In a first attempt, Post gives the following characterization of 'his' General Correspondence Principle:

Roughly speaking, this is the requirement that any acceptable new theory $L$ should account for its predecessor $S$ by 'degenerating' into that theory under those conditions under which $S$ has been well confirmed by tests. (Post 1971:

The General Correspondence Principle is claimed to be valid even across scientific revolutions. It presupposes that the predecessor theory $S$ and the successor theory $L$ "refer (in their statements) to at least some events or facts which are identifiably the same" (Post 1971: 220), or, to phrase it differently, that $S$ and $L$ share a common set of phenomena. The domain of $L$ is assumed to be larger than the domain of $S$ and the account given by $L$ will usually be more precise (or at least not less precise) than the account of the phenomena given by $S$. A typical example is the relation between classical mechanics and the special theory of relativity. The latter theory also correctly describes particles with a velocity close to the speed of light and provides a more accurate account at low velocities than the former.

Post goes on to discuss several possible relations between $S$ and $L$ that range from a complete reduction (which seems hardly ever to occur in science) to approximate or

\footnotetext{
${ }^{3}$ This section and the next draw on material published in Hartmann (2002).
} 
inconsistent correspondence, but without explanatory losses (such as the just mentioned relation between classical mechanics and the special theory of relativity). Other possible relations between $S$ and $L$ which exhibit losses would count as evidence against the General Correspondence Principle; Post holds that these relations never occured in the history of science of the last three hundred years - apart from one noteworthy exception that will be discussed below.

One of Post's favorite examples to support the General Correspondence Principle is the periodic system, which survived the quantum mechanical revolution. ${ }^{4}$ Post explains:

The periodic system is the basis of inorganic chemistry. This pattern was not changed when the whole of chemistry was reduced to physics, nor do scientists ever expect to see an explanation in the realm of chemistry which destroys this pattern. The chemical atom is no longer strictly an atom, yet whatever revolutions may occur in fundamental physics, the ordering of chemical atoms will remain. (Post 1971: 237)

Post generalizes this example and maintains that the low-level structure of theories is particularly stable, while higher and less-confirmed levels are subject to change in the process of scientific theorizing. The pattern of the atoms remains, although quantum mechanics replaced the former framework theory. This principle seems, at first sight, to be quite plausible; but is it correct? Doubts arise once one recalls that Post himself confesses that the successful part of $S$ may be smaller from the perspective of the new theory $L$ than from the perspective of $S$ (Post 1971: 232). Given this, it is not clear how there can be a 'resistant kernel' in the long run which "remains pragmatically true ... for all time", as da Costa and French (1993: 146) suggest.

Later Post refines his proposal to also account for theories $S$ and $L$ with a different vocabulary. These vocabularies have to be translated into each other and this translation $\mathcal{T}$ may turn out to be more difficult than a mere one-to-one mapping. Also, a condition $Q$ on $L$ has to be specified such that the truncated $L$ and $S$ have (perhaps only approximately) the same domain. If the well-confirmed part of $S$ is denoted by $S^{*}$ (the extent of which is only a conjecture at a given time ${ }^{5}$ ) the General Correspondence Principle can be conveniently expressed as $S^{*}=\mathcal{T}(L \mid Q)$ - the well-confirmed part of $S$ is identical to the suitably translated part of $L$ which fulfils the condition $Q$. If $L^{*}$ is the well-confirmed part of $L$ and $S^{* *}$ is the intersection of $S^{*}$ and $L^{*}$ then the thesis of zero Kuhn losses is that $S^{*}$ is identical to $S^{* *}$. Post claims that the historical record supports this thesis. ${ }^{6}$

\footnotetext{
${ }^{4}$ For an excellent account of this case study, see Scerri (2006).

${ }^{5}$ Cf. Koertge (1973: 172 f).

${ }^{6}$ For a comparison of Post's General Correspondence Principle with other correspondence principles, such as the ones suggested by Fadner, Krajewski, Radder, and Zahar see Radder (1991).
} 
It should be noted, however, that Post's analysis does not take the 'loser's perspective' into account. From this perspective there are indeed successes of the old theory which the new theory cannot account for. ${ }^{7}$ Besides, even from the 'winner's perspective' the thesis of zero Kuhn losses may be too strong. Saunders (1993: 296), for example, writes that "Laudan [(1981)] is right to insist that one can always find some theorem, deduction, conjecture, or explanation that has no precise correlate in the successor theory". He then goes on, though, to distinguish between significant and insignificant Kuhn losses; only the insignificant ones are, of course, 'allowed'. I will come back to this issue below. Radder (1991) has pointed out another problem for Post's approach: Not all equations of $L$ may 'degenerate' in equations of $S$. As an example, consider the famous formula $E=m_{0} c^{2}$ for the energy of a particle with rest mass $m_{0}$. This equation makes sense only in the special theory of relativity. It remains unaltered in the limit of low velocities $v$ (i.e. for $\beta:=v / c \rightarrow 0)$, although it does not correspond to an equation of classical mechanics.

According to Post, the General Correspondence Principle is both a descriptive and a normative thesis. It is considered to be a post hoc elimination criterion and theories which do not fulfill it should be, as Post boldly advises, consigned to the 'wastepaper basket' (Post 1971: 235). Examining cases from the history of science, Post only spotted one 'counterexample' to the General Correspondence Principle. Ironically it is the best theory we have today: quantum mechanics, a theory that, or so Post argues, does not account for the successes of its predecessor classical mechanics (Post 1971: 233). This is a crucial failure which Post blames on the supposed incompleteness of quantum mechanics (Post 1971: 234, 246). ${ }^{8}$ Quantum mechanics therefore does not, for Post, count as a case against the General Correspondence Principle. Instead the fact that quantum mechanics does not fulfil the General Correspondence Principle shows that this theory should not be accepted or at least that it should not be considered to be the successor of classical mechanics. It belongs, perhaps, in the wastepaper basket. Other proponents of a generalized correspondence principle, such as Radder, do not go as far and emphasize correspondence relations that do hold between quantum mechanics and classical mechanics. Their arguments will be examined in the next section.

Before doing so, another issue needs to be mentioned. So far, the following three theses are in conflict: (1) Post's General Correspondence Principle is descriptively correct, (2) the belief in the truth of quantum mechanics is justified and (3) quantum mechanics and classical mechanics share a common set of phenomena. Rather than rejecting theses (1) or (2) one might doubt thesis (3). Cartwright (1999), for example, argues that we

\footnotetext{
${ }^{7} \mathrm{Cf}$. Hoyningen-Huene (1993: 260-262) and the references to the work of Kuhn cited therein.

${ }^{8}$ It is interesting to speculate how Post would evaluate the recent work on decoherence and the alleged "emergence of a classical world in quantum theory". See Joos et al. (2003).
} 
have good reasons to believe that there are two disjunct classes of phenomena; some can be modeled by using the toolbox of quantum mechanics, others by relying on classical mechanics. There is consequently no quantum mechanical model of classical phenomena. Contrary to Cartwright, however, Post and - I believe - most physicists hold the view that quantum mechanics and classical mechanics do share a common set of phenomena. They assume that quantum mechanics accounts for the phenomena of classical mechanics in principle; it is merely a matter of computational complexity to demonstrate this. In the end, however, this might be nothing but a metaphysical dream.

What is the outcome of the discussion so far? First of all, when the General Correspondence Principle is applied, it often does not hold strictly, as Radder's example shows. Besides, there are losses from the loser's perspective and maybe also losses from the winner's perspective. Secondly, as a consequence of all this, there is a tension between the practice of actual science and a normative reading of the General Correspondence Principle. And yet Post is right when he points out that there is a lot of continuity in scientific theorizing, even across scientific revolutions. Still, the relations between various theories in the history of science are much more complicated than the General Correspondence Principle makes us believe. Perhaps there is no single and non-trivial principle which captures the rich structure and variety of developing scientific theories. This can only be established empirically. What is needed, therefore, is a careful examination of episodes from contemporary science and the history of science on which, perhaps, a meta-induction can be based. As a first step, it is helpful to highlight various relations which hold between successive scientific theories. This is what we will do in the next section.

\section{A Plurality of Correspondence Relations}

In the development of scientific theories, continuities as well as discontinuities appear. Hence, the interesting question to be addressed is this: Which elements of $S$ and $L$ correspond to each other, and which elements do not? Are there general rules that guide practising scientists in those difficult decision situations (if it can be reconstructed as such)? As a prolegomenon to such a task, it is reasonable to examine more closely how specific scientific theories relate to each other. Which elements are taken over, what are the motives for doing so and how are the elements of the old theory made to fit the new theory? Examining cases from various sciences, I will address these questions and provide a preliminary (and not necessarily exhaustive) list of correspondence relations which may hold between successive theories. Some theories exhibit more than one of these relations, and some correspondences appear at different stages of the development of a theory.

A first useful distinction is between ontological and epistemological correspondence rela- 
tions. An ontological correspondence relation holds between $S$ and $L$ if some or all of the entities of $S$ are also entities of $L$. In this contribution, I will consider only epistemological correspondence relations, i.e. relations between the theories in question. The following types of epistemological correspondence relations can be distinguished:

1. Term Correspondence. Here certain terms from $S$ are taken over into $L$. This is a standard strategy in the development of scientific theories. In The Structure of Scientific Revolutions Kuhn writes that "[s]ince new paradigms are born from old ones, they ordinarily incorporate much of the vocabulary and apparatus, both conceptual and manipulative, that the traditional paradigm had previously employed" (Kuhn 1996: 149). Now it is well-known that Kuhn also argues in the very same book that this continuity goes along with meaning variance and problems of reference. A standard example is the meaning shift from 'mass' in classical mechanics to 'mass' in the special theory of relativity. A disclaimer or two is in order here. Term correspondence does not imply that all terms of a theory correspond to terms in the successor theory. Often, only a few key terms are carried over, while others are left aside and new terms are coined in addition. Also, a correspondence relation between two theories can be established by a suitable translation of the respective terms. Term Correspondence is a rather minimal requirement; it is presupposed by all other corrrespondence relations to be discussed below.

2. Numerical Correspondence. Here $S$ and $L$ agree on the numerical values of some quantities (cf. Radder 1991: 203-204). Numerical Correspondence therefore presupposes Term Correspondence. An example is the spectrum of hydrogen in the Bohr model and in quantum mechanics. Although the assumptions that were made to calculate the spectrum differ considerably in both theories, they nevertheless lead to the same numerical values. Again, this is a rather weak kind of a correspondence relation which is moreover usually realized only approximately (as in the example just discussed). Its heuristic value is low since the principle can only be applied post hoc. Obviously, Numerical Correspondence is only interesting in the mathematical sciences; it does not apply, for instance, in large parts of biology or archeology.

3. Observational Correspondence. This kind of correspondence relation is introduced in Fine (1993) in the context of his interesting resolution of the quantum mechanical measurement problem. Fine does not accept Cushing's claim that Bohm's version of quantum mechanics should have been chosen according to Post's General Correspondence Principle (Cushing 1993: 262), because the Bohm theory "did not enable one to retrieve the classical and well-confirmed account of a ball rebounding elastically between two walls" (Fine 1993: 280). It therefore does not fulfil 
Post's correspondence principle. Bohm's theory does, however, fulfil a weaker form of a correspondence principle. Fine writes: "[W]here the classical account itself is well-confirmed, the Bohm theory 'degenerates' into the classical account of what we are expected to observe under well-defined conditions of observation" (Fine 1993: 280). Unfortunately, the standard Copenhagen version of quantum mechanics does not fulfil the principle of Observational Correspondence and Fine therefore presents his solution of the measurement problem in order to restore this. Abstracting from quantum mechanics, Observational Correspondence means that $L$ 'degenerates' into what we are expected to observe according to $S^{*}$ under well-defined conditions of observation. Observational Correspondence, like Numerical Correspondence, presupposes Term Correspondence, but differs from Numerical Correspondence, which may also apply when the quantities in question cannot be observed. Besides, Observational Correspondence relations can also hold in sciences which do not represent their content numerically. Observational Correspondence emphasizes the role of the conditions of observation which are especially important in the context of quantum mechanics. A heuristic principle based on the demand of Observational Correspondence is again only a post hoc selection criterion. It is of no help in the actual process of constructing new theories. Observational Correspondence alone also does not suffice to provide an explanation for the success of the old theory. It is therefore weaker than Post's General Correspondence Principle.

4. Initial or Boundary Condition Correspondence. According to a well-known view of scientific theories, a theory is a set of axioms (or laws) plus suitable initial or boundary conditions. Kamminga (1993) complains that the philosophical focus is too much on the axioms (or laws), leaving initial and boundary conditions aside. This is unfortunate, since especially in the non-formal sciences, Kamminga claims, these conditions play an important role which is relevant to the issue of inter-theory relations. It turns out that there are theories which incorporate consequences of their predecessor as an initial or boundary condition. An example from the research on the origin of life illustrates Kamminga's general point which she sums up as follows: "[I]n the attempt to integrate the original theory $T$ with another theory outside its domain, some consequence of the latter is incorporated into $T$ as an antecedent condition, which then places strong constraints on the selection of laws that have explanatory relevance in the modified theory $T^{\prime \prime}$ (Kamminga 1993: 77). This procedure, therefore, provides a link between the two theories. Note, however, that this way of connecting two theories is only a very loose one. It has some heuristic value but it should be noted that the assumptions taken over from the 
predecessor theory remain unexplained in the successor theory.

5. Law Correspondence. Laws from $S$ also appear in $L$. This kind of correspondence relation often holds only approximately. An example are the laws for the kinetic energy in classical mechanics and in the special theory of relativity. For low velocities, $T_{C M}=1 / 2 m v^{2}$ and $T_{S R T}=\left(m-m_{0}\right) c^{2}=1 / 2 m v^{2} \cdot\left(1+3 / 4 \beta^{2}+\mathcal{O}\left(\beta^{4}\right)\right)$ are approximately the same. Hence, the special theory of relativity reproduces and explains the successful part of classical mechanics. It is probably this kind of a correspondence relation which Post had in mind when he suggested his General Correspondence Principle. Law Correspondence implies Numerical Correspondence and presupposes Term Correspondence, the difficulties of which (such as meaning variance etc.) therefore occur again. Despite all this it is required that the terms in question have the same operational meaning in $S$ and $L$ (cf. Fadner 1985: 832). In many cases, Law Correspondence is only a post hoc selection criterion of theory choice. As Radder's above-mentioned example demonstrates, it may only hold for some of the laws of the theories in question.

6. Model Correspondence. This type of a correspondence relation comes in two variants. (1) A model which belongs to $S$ survives theory change and re-occurs in $L$. A typical example is the harmonic oscillator which is widely used in classical mechanics, but is also applied in quantum mechanics and in quantum field theory. It should be noted that models, such as the harmonic oscillator, are not only taken over by the theory which succeeds the original theory, but also by quite unrelated theories. This is best seen by pointing to all other theories of physics which employ the harmonic oscillator; in fact, it is difficult to find a theory which does not employ this model. Model Correspondence of this first kind has a considerable heuristic potential. It is, however, not guaranteed that the new theory explains the success of the old theory, because the model in question may be embedded in a completely new framework theory which may also affect the overall correspondence relation between $S$ and $L$. (2) Post mentions another strategy of theory construction which takes models seriously: "In this case we adopt a model already available which may initially have been offered as an arbitrary articulation of the formalism only. [...] It is a case of borrowing a model of the $S$-theory which contained features not essential for the modelling of the $S$-theory ('neutral analogy'), and assigning physical significance to such extra features" (Post 1971: 241). An example is the crystallographic models which were used already a century before physicists identified the units of the regular lattices with physical atoms. Sometimes, Post concludes, scientists built "better than they knew" (Post 1971: 242). This example also shows 
that Model Correspondence of this second kind may indeed lead to an explanation of the success of the predecessor theory. ${ }^{9}$ However, the criterion is highly fallible, as Post himself grants.

7. Structure Correspondence. Here the structures of $S$ and $L$ correspond. But what is a structure, and what does it mean that two structures correspond? One option is to use the term 'structure' only in its precise mathematical meaning. If one does so, it is not difficult to flesh out the idea of a correspondence relation between two structures by applying mathematical concepts such as sub-groups and group contractions. And indeed, many theories in physics can be linked to each other in this way. A typical example is the relation between the inhomogeneous Lorentz group (that characterizes the special theory of relativity) and the inhomogeneous Galilei group (that characterizes classical mechanics) which 'correspond' in a precise mathematical sense. In examples like this Structure Correspondence works best. Another interesting case is the relation between the theories of Ptolemy and Copernicus. Saunders shows that "[a]n astronomy based only on epicycles [...] corresponds to an expansion of the form $\sum_{i} c_{i} \exp \left(\mathrm{i} \omega_{i} t\right)$ (with the earth chosen as origin)" (Saunders 1993: 299). So the mathematical structures of both theories are (perhaps only approximately) the same, which leads Saunders to the conclusion that there is no reason to worry about the abandonment of the Aristotelian world-view or a wholesale change of paradigm (Saunders 1993: 298).

Saunders' large-scale fight against relativism ${ }^{10}$ appears somewhat cheap; parts of theories where problems for the idea of correspondence show up are stamped as 'insignificant' (such as the ontology of a theory ${ }^{11}$, but also laws) while only the mathematical structure of a theory remains, in some sense, stable. But even here things are not that easy. With respect to the role of gravity, Saunders concedes that he does "not suggest that these things can be completely codified" but goes on to confess that this strategy "is, and [...] has always been, the essence of the enterprise of dynamics" (Saunders 1993: 306). Confessions like this are not enough to provide a vigorous defence of the cumulative, progressive view of the history of physics, but Saunders showed that mathematical structures of consecutive theories may and often do correspond in a strict mathematical sense.

Structure Correspondence does not imply Numerical Correspondence. Often, the

\footnotetext{
${ }^{9}$ For more on the relation between models and theories see Frigg and Hartmann (2006).

${ }^{10}$ Note that for Saunders, 'relativism' is a collective name for social constructivism, historicist epistemology, linguistic holism and anti-realism; cf. Saunders (1993: 295 f).

${ }^{11}$ Cf. Saunders' discussion of ether in Saunders (1993: 299).
} 
structure is 'too far away' from the empirical basis of a theory in order to guarantee continuity at that level (especially in the cases Saunders has in mind). It is therefore not at all trivial to reproduce the empirical success of the precursor theory once one has decided to take over parts of the structure of the old theory. Despite this, Structure Correspondence has a very high heuristic value, especially in contemporary theoretical physics. Because of the huge gap between the respective theories (such as superstring theory) and the world to which we have empirical access, structure-based reasoning, such as symmetry considerations, is often the only tool which enables scientists to proceed. However, when it comes to other sciences, such as biology or archeology, Structure Correspondence does not seem to be of much value, especially if one explicates 'structure' mathematically.

Three conclusions can be drawn from the above analysis: First, successive theories can be related in many ways. Sometimes only Numerical Correspondence holds (at least approximately), in other cases entire mathematical structures correspond. Every case is different. This is why global philosophical issues, such as meaning variance and incommensurability, should be discussed 'locally', i.e. on the basis of concrete case studies that exemplify specific types of relations between scientific theories. The details might, and often do, matter.

Second, there are continuities and discontinuities in scientific theorizing, although it is not a priori clear which elements of a theory will survive theory change, and which ones will have to go. An additional difficulty for correspondence theorists is the notorious problem of underdetermination. Maybe there is no unique best choice regarding which elements of successive theories should correspond and which should not correspond with each other.

Third, the philosophical project of a methodology is best described by the picture of a toolbox. According to this view, methodologists extract - ideally on the basis of a wealth of case studies - a set of methods and techniques which can tentatively be applied by practicing scientists in a particular situation. What is in the toolbox may, however, depend on time. Good scientists know, of course, already a wealth of tricks and methods, and they also know how to use them flexibly and appropriately. This view of the status of methodology is a middle ground between two extreme positions. Zahar (1983: 258 f) defends a rather strong form of a rational heuristics which leaves little room to chance and other influences, while Popper's (1972: ch. 7) evolutionary picture supports the opposite view, that there is no rational heuristics and it is the job of the scientists to make bold conjectures which then have to 'survive' empirical tests and rational criticism (cf. Radder 1991: 201 f). My conclusion seems, after all, to be similar to Post's view 
on the role of heuristics which he illustrates with an apt analogy: "The study of the structure of existing houses may help us in constructing new houses" (Post 1971: 217).

\section{Modelling High-Temperature Superconductivity: A Case Study}

In this section, I will look at a case study from contemporary physics and ask which role heuristic principles such as the General Correspondence Principle play in science. The case study deals with a scientific episode that is ongoing. So far, there is no consensus in the scientific community, only a multitude of more or less elaborated competing research programs and strategies. I'll identify some of these strategies and ask which role correspondence considerations play when scientists are confronted with an intricate problem.

The case study deals with our theoretical understanding of high-temperature superconductivity. Conventional superconductivity is a phenomenon long well known and understood. It occurs at extremely low temperatures close to the absolute zero. For a long time, it was considered to be impossible to find or produce substances that remain superconducting if the temperature is raised to, say, $30 \mathrm{~K}$ or more. The breakthrough occurred in 1986 with the work of Georg Bednorz and Alex Müller, who discovered the first high-temperature superconductor LBCO with a transition temperature of $35 \mathrm{~K}$ (Bednorz and Müller 1988). Later, materials with a transition temperature of up to $160 \mathrm{~K}$ were produced. It turned out that the materials that are superconducting at such high temperatures are very special: they have a layered structure made up of one or more copper-oxygen planes and exhibit an abnormal behavior also when in the normal state. This complicates the theoretical understanding of these so-called cuprates considerably and so it is no surprise that, despite a lot of theoretical work over the last twenty years and a wealth of experimental data, no theoretical understanding of high-temperature superconductivity is forthcoming. ${ }^{12}$

There is, however, a well-confirmed theory of conventional superconductors. This theory, the so-called BCS theory - named after its inventors James Bardeen, Leon Cooper and Robert Schrieffer -, is a microscopic theory that explains the appearance of a superconducting phase by showing how bound pairs of electrons with opposite spin and momentum are formed if the temperature is below the critical temperature. Although the (fermionic) electrons in a so-called Cooper pair are often separated from each other

\footnotetext{
${ }^{12}$ See Tinkham (1996) and Waldram (1996) for (somewhat dated) overviews. More recent reviews are Anderson (2006) and Kivelson (2006).
} 
by a large distance, they act effectively as a single particle which has the quantum statistical properties of a boson. And this is why a large number of Cooper pairs can be in the lowest energy state, which in turns leads to the vanishing of the electrical resistance in the materials. Complementing the BCS theory, Bardeen, Cooper and Schrieffer proposed a specific model - the BCS model - that specifies the concrete mechanism that is responsible for the creation of Cooper pairs. This mechanism is based on so-called $s$-wave interactions of the electrons, mediated by the vibrating crystal lattice, and accounts for all phenomena involving superconductivity discovered until $1986 .^{13}$

When it comes to understanding high-temperature superconductivity, two points are uncontroversial: (i) The BCS theory also applies, i.e. high-temperature superconductivity results from the formation of electron pairs at temperatures below the (materialdependent) critical temperature. (ii) The BCS model does not apply. More specifically, it is generally accepted that $s$-wave interactions cannot account for the extremely high transition temperature that we find in the cuprates. And so the task is to develop a new model. To do so, physicists follow a wide variety of approaches that can be located on a spectrum ranging from conservative to highly revolutionary apoproaches.

Conservative approaches aim at developing an account that deviates as little as possible from the theoretical framework of the BCS theory (i.e. the Fermi liquid theory) and the more specific assumptions of the BCS model. Revolutionary approaches attempt to formulate an account of high-temperature superconductivity in a new theoretical framework and propose mechanisms that deviate considerably from the BCS model. While some authors suggest different mechanisms for different types of materials, others want to identify the mechanism of high-temperature superconductivity. Besides these extreme approaches, a whole range of approaches in between has been put forward.

All of these approaches are developing, constantly modified and occasionally completely rejected, but none has yet succeeded. Even twenty years after the astonishing discovery of Bednorz and Müller, there is no satisfactory and generally accepted theory of hightemperature superconductivity. Given that we had to wait 46 years from the discovery of conventional superconductivity (by Heike Kamerlingh Onnes in 1911) to the formulation of the BCS theory (in 1956), we might have to be patient for quite a while. That it is taking so long is seen as an indication that a major theoretical breakthrough can be expected. In situations like this, much is at stake and the debate among the members of the relevant scientific community often touches philosophical and methodological issues. So let us have a look at some of the proposals that are currently discussed:

1. The conservative strategy

\footnotetext{
${ }^{13}$ For technical details, see Tinkham (1996: ch. 3).
} 
The proponents of a conservative research strategy (such as David Pines and David Scalapino) propose rather minimal modifications of the BCS model in order to account for the phenomenon of high-temperature superconductivity. They keep, for example, the Fermi liquid theory as a theoretical framework. What is replaced, in a minimal way, is the specific pairing mechanism. Instead of $s$-wave interactions among the electrons, the next more complicated interactions, mediated by $d$-waves (plus spin fluctuations), are considered. $d$-waves are suggested by the spatial structure of the substances in question and so have an empirical foundation (Scalapino 1995). Supporters of this approach (and related approaches) point to its successes when it comes to understanding experiments. They also stress the minimal character of the modifications on the BCS model that have to be made.

Critics (such as Philip Anderson) object that the spin-fluctuation theory is not a real theory, but just a bunch of heuristic tools with various unjustified assumptions. It also counts against the spin-fluctuation theory, or so Anderson claims, that it can only be applied by using high-powered computer simulations - a procedure which cannot be controlled independently. Supporters reply that the complexity of the problem suggests that it is treated with such methods. ${ }^{14}$

More recently, a peculiar charge ordering pattern in two separate states and regimes of the cuprates has been discovered. To explain these patterns, S. C. Zhang used the crystalline ordering of the $d$-wave Cooper pairs and showed how this new state of matter fits into the global phase diagram of cuprates with a high transition temperature. He also derived several predictions from his theory, which appear to have recently been verified in transport measurements. Indeed, in the last decade the experiments showed more clearly that the cuprates with a high transition temperature have a very complex phase diagram with many competing phases. In optimally and underdoped materials, the state of matter out of which the superconductivity arises exhibits a so-called pseudogap at temperatures which are high compared to the transition temperature (Norman et al. 2005). The pseudogap was found only three years after Bednorz and Müller's original discovery, but its physical origin, its behavior and whether it constitutes a distinct phase of matter is still not well understood. What is clear, however, is that the pseudogap will play an important role in any theory of high temperature superconductivity. Lee (2006), for example, believes that the existence of the pseudogap supports the Charge Density Wave (CDW) theory as the large pseudogap in the cuprates can be generated by a CDW

\footnotetext{
${ }^{14}$ See Anderson (1995), Anderson and Schrieffer (1991), Anderson and Mott (1996) and Ford and Saunders (1997).
} 
order with $d$-symmetry.

2. The revolutionary strategy

Philip Anderson started off defending a truely revolutionary strategy. He submited that the problem of understanding high-temperature superconductivity cannot be attacked by a minimal modification of the BCS model. He hence proposed to give up a standard assumption in solid state physics - that the system can be described as a Fermi liquid - and to replace it with the assumption that the systems in question are, in the normal as well as in the superconducting state, so-called Luttinger liquids. Anderson's account was highly speculative and was rejected by most physicists. ${ }^{15}$ Meanwhile, in the light of new experimental evidence, Anderson gave up his original account as well and proposed a new one with a somewhat different flavor (Anderson et al. (2004)). This new account is based on an interesting analogy between a Mott insulator and high-temperature superconductivity and is sometimes called a theory of a doped Mott insulator. Though less revolutionary than the old one, Anderson's new account is fundamentally different from other approaches that are mostly based on perturbative many-body theory. During the last two years, it has attracted considerable attention amongst both theorists and experimentalists. However, it is still far from being generally accepted.

And so the question remains how conservative or revolutionary a future theory of hightemperature superconductivity should be. As there is no consensus in the scientific community over which strategy is the right one, a final assessment of the various strategies is not possible at the moment. While a majority of physicists seem to be in favour of a conservative account, Anderson's (1995: 38) reply is still worth taking seriously: "[I]f it is time for a revolution, enjoy it and relax!"

\section{Correspondence at Bay? - Some Lessons}

What are the implications of our case study for the General Correspondence Principle? First, it has to be noted that the starting point for the development of theories of hightemperature supercondictivity was not an internal anomaly of the predecessor theory, i.e. the BCS theory (and the BCS model) of conventional superconducters. Quite to the contrary, the account given by BCS turned out to be an excellent tool to learn about the physics (and chemistry) of superconductors for many years. No one doubted, for example, that it is a perfectly consistent theory. And yet after a while, new experimental

\footnotetext{
${ }^{15}$ See Anderson (1997) for a book-length exposition of his approach and Leggett (1997) for a criticism.
} 
data were produced that could not be fit into the BCS account. ${ }^{16}$ This is what prompted the current developments.

Second, none of the proposals for a theory of high-temperature superconductivity that have been for put forward so far contain the BCS account as a limiting case. There is, hence, no new theory that accounts for the successes of the old theory as well as for the class of new phenomena. And so the General Correspondence Principle seems to be violated. Or isn't it? A way out for the correspondence theorist is to argue that the General Correspondence Principle cannot be applied to the case of high-temperature superconductivity. She could argue that the corresponding materials belong to a completely different class of materials, that the phenomena in question (i.e. conventional and high-temperature superconductivity) are too different, and that the similarity between them is at best superficial. And so we are not supposed to apply the General Correspondence Principle (or something like it). What is at stake is the question of what the domain of applicability of methodological principles such as General Correspondence Principle is and how we find or identify this domain. I have no principled answer to this question (and doubt that there is one). At best, I think, we can trust the judgement of the scientific community, which is the only authority I can see. For now, the scientific community has not made up its mind on high-temperature superconductivity, and the field is characterized by a large amount of dissent over questions ranging from specific scientific questions to methodological questions.

Looking more closely at the various proposals for a theory of high-temperature superconductivity, one realizes that all of them take over some elements of the BCS account. All of them, for example, adopt the idea that pairing is responsible for superconductivity. ${ }^{17}$ And some of them modify the BCS pairing mechanism only slightly, so that the mechanism responsible for high-temperature superconductivity can be understood as a natural extension of the mechanism for conventional superconductivity. I conclude from this that there is certainly some continuity on the theoretical level. But continuity is not an allor-nothing matter (as the General Correspondence Principle makes us think). How far the continuity reaches (or should reach) will depend on the specific case in question and is a matter of debate. In the course of science, different (and sometimes even the same) scientists will try out different approaches and adopt different general methodological or scientific principles. The best way to understand this, as already argued at the end of section 3 , is to consider methodological principles as tools that are tentatively adopted by some scientists for a specific purpose. Whether they are good tools or bad tools depends on the specific case and will be decided, in the end, by the scientific community.

\footnotetext{
${ }^{16}$ The BCS account is the BCS theory plus the BCS model.

${ }^{17}$ For a recent discussion, see Tsai and Kivelson (2006).
} 
The General Correspondence Principle is just one tool among many that scientists tentatively use in the process of theory (or model) construction. In many cases, there will be a lot of dissent over the question of how closely the successor theory should be related to its predecessor. The respective considerations are typically subtle and deeply entangled with the problem at hand. I submit that general methodological principles unfold their fruitful action only when adapted to a specific problem.

This raises a difficulty for those philosophers of science who are interested in the identification of general methodological principles. Instead of doing this, it might be best to restrict oneself and help put together a toolbox of principles that scientists can tentatively use on an as-needed basis. To do this seems to me to be an important task. It is both doable - unlike the program of rational heuristics - and worthwhile. Philosophers of science are especially qualified to undertake such a project, as they overlook a greater part of science than do practitioners, who are often confined between the boundaries of their field of specialization.

\section{Rationality, Realism and Coherence}

Even if the continuity that we observe in scientific theorizing does not fit into the neat picture that goes with the Generalized Correspondence Principle, there is no doubt that there is a lot of continuity (or stability, as Hacking puts it) in scientific theorizing. So the following questions arise: Why is it that there is so much continuity in science? And how can this continuity be understood philosophically? Note that these are questions that cannot be attacked locally, i.e. on the basis of case studies alone.

In "The Social Construction of What?", Hacking (1999) presents two explanations for the prevelent continuity in scientific theorizing. The first explanation, realism, stresses factors internal to science and comes in various variants. One of them is convergent realism, which holds that successive theories of 'mature science' approximate the truth (i.e. the ultimate or final theory) better and better. This presupposes the existence of a measure of the distance of a given theory from the truth (or at least an ordering relation), which is a controverial topic despite all the worthwhile work on verisimilitude and truthlikeness ${ }^{18}$, and conflicts with the many discontinuities that emerged in the development of 'mature' scientific theories, as Laudan (1981) has convincingly demonstrated. Another problem of convergent realism is that there might be no ultimate theory. It is possible that the process of constructing ever better theories never ends because "there are infinitely many levels of structure that can be unpacked, like an infinitely descending sequence of Chinese

\footnotetext{
${ }^{18}$ See Niiniluoto (1999) for a recent exposition.
} 
boxes, or to use the more colloquial expression: it is boojums all the way down" (Redhead 1993: 331).

A weaker variant of realism, which seems to be able to handle the problems raised by Laudan and others, is structural realism. According to (one version of) this position, at least the high-level mathematical structures of scientific theories converge. Continuity on the level of ontology and perhaps even on the level of one or another law is not required. However, my discussion in the previous section suggests that there is not enough "empirical evidence" to support this position. Besides, there are more plausible ways to explain the persistence of certain mathematical structures; I will come back to this below. ${ }^{19}$

The second explanation discussed by Hacking, constructionism, comes in several variants as well. All of them emphasize the role of factors external to science. For example, in the previously-mentioned section on stability, Hacking quotes the historian of science Norton Wise, who argues that culture and science are inseparably connected with each other. Cultural influences go into the discovery of scientific theories and leave an indelible footprint there. Steven Weinberg, whom Hacking quotes approvingly, argues, however, that these influences "have been refined away" (Hacking 1999: 86).

Adopting a Kuhnian line of thought, a constructionist can explain the remarkable stability of scientific theories as follows: she reminds us that scientists grow up and get trained in a certain research tradition, they learn all the theories and techniques of this tradition and, of course, they want to promote their career. These scientists are well-advised to demonstrate their affiliation to the tradition in question by contributing to the corresponding research program. All too radical junior-scientists are not protegéed and their careers may take a turn for the worse. After all, the scientific community does not reward disloyal behaviour. Here is another, and perhaps somewhat more plausible, explanation of the continuity in scientific theorizing by external factors: It is simply too costly to start from scratch when confronted with a new scientific problem. Scientists who do so will not be able to produce a sufficient number of publications in renowned journals which is essential to carry on with academia. This also explains why the high-level structure of theories is so stable: since so much depends on it, a revision or replacement would be very costly indeed. Although there is some undeniable truth to these stories, I think that there is more to be said.

I conclude that both explanations, realism and constructionism, do not suffice to account for the prevalent continuity of scientific theorizing. Interestingly, they do not account for the occasional discontinuities (or revolutions, as Hacking puts it) in scientific theorizing

\footnotetext{
${ }^{19}$ Another realist way put forward to account for the stability of scientific theories is Radder's (1988, 1991) moderate realism.
} 
as well. Clearly, discontinuities do not fit in the scheme of convergent realism. But also weaker forms of realism do not explain the relative weights of continuities and discontinuities in scientific theorizing. And constructionism fails to do so as well. However, it is the (possibly case-dependent) relative weights that cry out for an explanation and so I conclude that an alternative explanation is needed that helps us understand why there is as much continuities and as much discontinuities in scientific theorizing as there is. Such an explanation should fulfill the following three conditions. (i) It should be sufficiently general, as our goal is a philosophical understanding of the scientific practice. (ii) It should be descriptively correct and account for successful scientific practice. (iii) It should combine internal and external factors, as both seem to be important.

Providing an explanation of the relative weights of continuities and discontinuities in scientific theorizing that fulfills these three conditions is a difficult task and an easy answer such as 'realism' or 'constructionism' is unlikely to succeed. Instead I suggest to adopt a formal philosophical framework and construct philosophical models within this framework, mimicking the successful scientific methodology of constructing models of a theory mentioned in the Introduction. The philosophical framework will be general and satisfies condition (i). The models will provide details to account for more specific cases, which helps satisfying condition (ii). Constructing more specific models makes sense as the relative weights will depend on the specific scientific theories in question. The framework we chose will make sure that internal and external factors are taken into account, thus satisfying condition (iii). Let us now see how this works.

The central idea of my own explanation, coherentism, is that the transfer of elements of the predecessor theory $S$ (whose well-confirmed part $S^{*}$ is non-empty) into the successor theory $L$ increases the overall coherence of our knowledge system. The word "coherence" is here used in the epistemological sense, i.e. as a measure for how well a body of beliefs "hangs together". As Lawrence BonJour explains, "[it] is reasonably clear that this 'hanging together' depends on the various sorts of inferential, evidential, and explanatory relations which obtain among the various members of a system of belief, and especially on the more holistic and systematic of these." (BonJour 1985: 93) Given this account of coherence, it is plausible that $S$ (or better: $S^{*}$ ) and $L$ cohere more if, ceteris paribus, the two theories share common elements.

Before working out my coherentist account, a potential objection has to be addressed. One may ask why both $S$ (or $S^{*}$ ) and $L$ should be in our knowledge system at the same time. Why isn't $S$ simply abandoned and replaced by a theory $L$ which is itself more coherent than the conjunction of $S^{*}$ and $L$ ? Although some theories in the history of science were indeed abandoned altogether and no elements were transfered from $S$ to $L$ (such as the phlogiston theory), this is often (and especially with the theories of the 
last 150 years $^{20}$ ) not the case. Theories such as classical mechanics are still successfully applied, although this theory is, strictly speaking, superseded by quantum mechanics and Einstein's theories of relativity. There are indeed a host of reasons why we still work with (the well-confirmed part of) classical mechanics. Most importantly, perhaps, is that classical mechanics has the practical advantage that it can be applied to problems where, for instance, quantum mechanical calculations are not feasible (and would presumably give the same result anyway). There is, however, also an epistemic reason why classical mechanics is still so popular. The theory provides explanations that quantum mechanics is unable to provide. This is, again for practical reasons, because classical mechanics can account for phenomena quantum mechanics cannot account for. However, we also favor the Newtonian explanation of, say, the tides over an explanation based on Einstein's general theory of relativity as the concepts classical mechanics employs (such as force) are "closer" to the phenomenon in question which arguably helps us to understand. An explanation of the tides based on the properties of curved spacetime would only obscure the situation. Good explanations function like this, and as it is one of the goals of the scientific enterprise to provide explanations of the phenomena in its domain, (the well confirmed part of) classical mechanics is indispensable and remains an integral part of our knowledge system.

Let us now develop our tentative account of scientific theory change. To do so, we need (i) a precise account of the notion of coherence and how it is measured. To measure the coherence of a scientific theory (or of a set of scientific theories), we also need (ii) a representation of a scientific theory that suits this request. As we will see, choosing a Bayesian framework will satisfy both requests.

We explicate (the empirical part of) a scientific theory $T$ as a set of interrelated models $\left\{M_{1}, \ldots, M_{n}\right\}$. Here a model is represented by a proposition $M_{i}$ which may itself be a conjunction of more elementary propositions (such as instantiations of laws, additonal model assumptions and initial and boundary conditions). Each model $M_{i}$ is related to a phenomenon, represented by a proposition $E_{i}$ (for "evidence"), that it accounts for. We define a probability distribution $P$ over all model variables $M_{i}$ and all phenomena variables $E_{i}$. This probability distribution is best represented by a Bayesian Network. In such a network, there is an arrow from each model node $M_{i}$ to its corresponding phenomenon node $E_{i}$, and there are various arrows between the model nodes which reflects the idea that the models in a scientific theory mutually support each other. By doing so, BonJour's "various sorts of inferential, evidential, and explanatory relations which obtain among the various members of a system of belief" can be modeled probabilistically. For example, if model $M_{2}$ entails model $M_{1}$, then $P\left(M_{1} \mid M_{2}\right)=1$, and if model $M_{2}$ supports

\footnotetext{
${ }^{20}$ Cf. Fahrbach (forthcoming).
} 
model $M_{1}$, then $P\left(M_{1} \mid M_{2}\right)>P\left(M_{1}\right)$ (for details, see Hartmann (2007)).

In the next step, these probabilistic dependencies between models have to be aggregated to arrive at a coherence measure $\operatorname{coh}(T)$ of the theory $T$. While there are several different proposals in the literature for how to do this, my preferred one is laid out in my book Bayesian Epistemology (with Luc Bovens). In this book, we show that no such coherence measure exists and that all that can be done is the specification of a function that generates a partial ordering over a set $\mathcal{T}=\left\{T_{1}, \ldots, T_{n}\right\}$ of theories. Sometimes there is no fact of the matter, which of two theories is more coherent. But often there is.

At this point, the natural question arises why coherence is a good thing. Even if scientists do aim for a highly coherent knowledge system (which explains the use of correspondence considerations), it is not clear why science should aim for a highly coherent knowledge system. So what is at stake is the normative question. Simply put, the answer is that, given certain conditions, coherence is truth-conducive. ${ }^{21}$ In the Bayesian framweork, this means that the more coherent set of, say, models, is also the one that has the greater posterior probability (provided that certain ceteris paribus conditions are satisfied). This is an important result as it makes informal discussions of the coherence theory of justification more precise (Bovens and Hartmann (2003a, b)).

Similarly, the following can be proven: If the new theory $L$ adds one additional model to its predecessor $S^{*}$ and if $L$ is more coherent than $S^{*}$, then $L$ has a higher posterior probability than $S^{*}$ if the prior probability of $L$ is not much lower than the prior probability of $S^{*}$. For details and a proof, see Hartmann (in preparation). Note that there is clearly a correspondence relation between $S^{*}$ and $L$ as all successes (as well as the failures) of the old theory are taken over in the new, more encompassing theory.

What I have presented are the initial steps of a philosophical research program. Clearly much more needs to be said and more philosophical models have to be studied to better evaluate the prospects of a full-fledged Bayesian account of scientific theory change. For example, a Bayesian account of the various correspondence relations discussed in sec. 3 has to be given. If all goes well, we have a methodology at hand that will help us to explain (using the notion of coherence) and justify (using the truth-conduciveness of coherence) the successful scientific practice. But we have to be cautious and not expect too much. As Earman (1992: ch. 8) points out, scientific theory change presents a whole range of other problems for the Bayesian. So I do not pretend that all aspects of scientific theory change can be accounted for in Bayesian term. However, until the contrary is proven, I follow Salmon (1990) and take Bayesianism to be an attractive philosophical research

\footnotetext{
${ }^{21}$ I here follow the standard usuage of the term. Note, however, that "truth-conducive" is a misnomer as Bayesians only specify subjective probabilities, and a posterior probability of 1 does not entail the truth of the proposition in question. I thank Kevin Kelly for pointing this out to me.
} 
program that helps us to illuminate the intricacies of scientific theory change.

Before closing, a word is in order about the nature of the probabilities in our Bayesian framework. Bayesians typically assume that individual agents have subjective degrees of belief which I take to include internal as well as external factors. These degrees of belief may differ from scientist to scientist as different scientists may have different background knowledge and different "scientific tastes". And yet, we observe that different scientists often assess new hypotheses similarly and reach similar conclusions about their acceptability. There is much to Kuhn's idea that the scientific community is the real decision-making agent, and it is an interesting research project to construct models for the aggregation of individual probability functions to a probability function of the scientific community. I have to leave this task for another occasion.

\section{Acknowledgement}

I am indebted to Léna Soler and Jan Sprenger for excellent comments and suggestions, to Clark Glymour for very useful discussions and feedback, and to Maria Elena Di Bucchianico for help with the case study.

\section{References}

Anderson, P. and R. Schrieffer (1991). 'A Dialog on the Theory of High- $T_{c}$ ', Physics Today, June, 55-61.

Anderson, P. (1995). 'Condensed Matter: The Continuous Revolution', Physics World 8, December, 37-40.

Anderson, P. and N. Mott (1996). 'High-Temperature Superconductivity Debate Heats Up', Physics World 9, January, 16.

Anderson, P. (1997). The Theory of Superconductivity in the High-T $T_{c}$ Cuprates. Princeton: Princeton University Press.

Anderson, P., P. Lee, M. Randeria, T. Rice, N. Trivedi and F. Zhang (2004). 'The Physics Behind High-Temperature Superconducting Cuprates: The 'Plain Vanilla' Version of RVB', Journal of Physics - Condensed Matter 16 (24), R755-R769.

Anderson, P. (2006). 'Present Status of the Theory of the High- $T_{c}$ Cuprates', Low Temperature Physics 32 (4-5), 282-289. 
Bednorz, J. G. and K. A. Müller (1988). 'Perovskite-Type Oxides - The New Approach to High- $T_{c}$ Superconductivity', Reviews of Modern Physics 60, 585-600.

BonJour, L. (1985). The Structure of Empirical Knowledge. Cambridge, Mass.: Harvard University Press.

Bovens, L. and S. Hartmann (2003a). Bayesian Epistemology. Oxford: Oxford University Press.

Bovens, L. and S. Hartmann (2003b). 'Solving the Riddle of Coherence', Mind 112, 601-634.

Cartwright, N. (1999). The Dappled World: A Study of the Boundaries of Science. Cambridge: Cambridge University Press.

Cushing, J. (1993). 'Underdetermination, Conventionalism and Realism: The Copenhagen vs. the Bohm Interpretation of Quantum Mechanics', in: French and Kamminga (1993), 261-278.

da Costa, N. and S. French (1993). 'Towards an Acceptable Theory of Acceptance: Partial Structures and the General Correspondence Principle', in: French and Kamminga (1993), 137-158.

Earman, J. (1992). Bayes or Bust? An Examination of Bayesian Confirmation Theory. Cambridge, Mass.: MIT Press.

Fadner, W. (1985). 'Theoretical Support for the Generalized Correspondence Principle', American Journal of Physics 53 (9), 829-838.

Fahrbach, L. (forthcoming). 'The Pessimistic Meta-Induction and the Exponential Growth of Science'. Unpublished manuscript. University of Konstanz.

Fine, A. (1993). 'Measurement and Quantum Silence', in: French and Kamminga (1993), 279-294.

Ford, P. and G. Saunders (1997). 'High Temperature Superconductivity - Ten Years On', Contemporary Physics 38, 63-81.

French, S. and H. Kamminga (eds.) (1993). Correspondence, Invariance and Heuristics. Essays in Honour of Heinz Post. Dordrecht: Kluwer.

Frigg, R. and S. Hartmann (2006). 'Models in Science', Stanford Encyclopedia of Philosophy (Spring 2006 Edition). 
Hacking, I. (1999). The Social Construction of What? Cambridge, Mass.: Harvard University Press.

Hartmann, S. (2002). 'On Correspondence', Studies in History and Philosophy of Modern Physics 33B, 79-94.

Hartmann, S. (2007). 'Modeling in Philosophy of Science', to appear in a Festschrift for Patrick Suppes (ed. by W.K. Essler and M. Frauchiger). Frankfurt: ontos-Verlag.

Hartmann, S. (in preparation). 'Normal Science and its Justification'.

Hoyningen-Huene, P. (1993). Reconstructing Scientific Revolutions: Thomas S. Kuhn's Philosophy of Science. Chicago: The University of Chicago Press.

Joos, E. et al. (2003). Decoherence and the Appearance of a Classical World in Quantum Theory. Berlin: Springer.

Kamminga, H. (1993). 'Taking Antecedent Conditions Seriously: A lesson in Heuristics from Biology', in: French and Kamminga (1993), 65-82.

Kivelson, S.A. (2006). 'Superconductivity on the Verge of Catastrophe', Nature Materials 5 (5), 343-344.

Koertge, N. (1973). 'Theory Change in Science', in: G. Pearce and P. Maynard (eds.): Conceptual Change. Dordrecht: Reidel, 167-198.

Kuhn, T. S. (1996). The Structure of Scientific Revolutions. Chicago: The University of Chicago Press.

Laudan, L. (1981). 'A Confutation of Convergent Realism', Philosophy of Science 48, $19-49$.

Lee, D.H. (2006). 'D-Symmetry CDW, Fermi Arcs, and Pseudogap in High $T_{c}$ Cuprates', talk presented at The 8th International Conference on Materials and Mechanisms of Superconductivity and High Temperature Superconductors. Dresden, 9-15 July 2006.

Leggett, T. (1997). 'Superconducting Thoughts Meet Sceptical Resistance', Physics World 10, October, 51-52.

Niiniluoto, I. (1999). Critical Scientific Realism. Oxford: Oxford University Press.

Norman M., D. Pines and C. Kallin (2005). 'The Pseudogap: Friend or Foe of High $T_{c}$ ?', Advances in Physics 54 (8), 715-733. 
Popper, K. (1972). Objective Knowledge. An Evolutionary Approach. Oxford: Clarendon Press.

Post, H. (1971). 'Correspondence, Invariance and Heuristics', Studies in History and Philosphy of Science 2, 213-255. Reprinted in French and Kamminga (1993), 1-44.

Radder, H. (1988). The Material Realization of Science. Assen: Van Gorcum.

Radder, H. (1991). 'Heuristics and the Generalized Correspondence Principle', British Journal for the Philosophy of Science 42, 195-226.

Redhead, M. (1993). 'Is the End of Physics in Sight?', in: French and Kamminga (1993), $327-341$.

Salmon, W.C. (1990). 'Rationality and Objectivity in Science, or Tom Kuhn Meets Tom Bayes', in: C. W. Savage (ed.), Scientific Theories. Minneapolis: University of Minnesota Press, 175-204.

Saunders, S. (1993). 'To What Physics Corresponds', in: French and Kamminga (1993), $295-325$.

Scalapino, D. (1995). 'The Case For $d_{x^{2}-y^{2}}$ Pairing in Cuprate Superconductors', Physics Reports 250, 329-365.

Scerri, E. (2006). The Periodic Table: Its Story and Its Significance. New York: Oxford University Press.

Tinkham, M. (1996). Introduction to Superconductivity. New York: McGraw-Hill.

Tsai, W. and S. Kivelson (2006). 'Superconductivity in Inhomogeneous Hubbard Models', Physical Review B 73 (21), 214510.

Waldram, J. (1996). Superconductivity of Metals and Cuprates. Bristol: Institut of Physics Publishing.

Zahar, E. (1989). Einstein's Revolution: A Study in Heuristic. La Salle, Ill.: Open Court.

Center for Logic and Philosophy of Science

Tilburg University

5000 LE Tilburg

The Netherlands 